\title{
Approaches for Achieving Broadband Achromatic Phase Shifts for Visible Nulling Coronagraphy
}

\author{
Matthew R. Bolcar* and Richard G. Lyon \\ NASA Goddard Space Flight Center, 8800 Greenbelt Rd., Greenbelt, MD 20771
}

\begin{abstract}
Visible nulling coronagraphy is one of the few approaches to the direct detection and characterization of Jovian and Terrestrial exoplanets that works with segmented aperture telescopes. Jovian and Terrestrial planets require at least $10^{-9}$ and $10^{-10}$ image plane contrasts, respectively, within the spectral bandpass and thus require a nearly achromatic $\pi$-phase difference between the arms of the interferometer. An achromatic $\pi$-phase shift can be achieved by several techniques, including sequential angled thick glass plates of varying dispersive materials, distributed thin-film multilayer coatings, and techniques that leverage the polarization-dependent phase shift of total-internal reflections. Herein we describe two such techniques: sequential thick glass plates and Fresnel rhomb prisms. A viable technique must achieve the achromatic phase shift while simultaneously minimizing the intensity difference, chromatic beam spread and polarization variation between each arm. In this paper we describe the above techniques and report on efforts to design, model, fabricate, align the trades associated with each technique that will lead to an implementations of the most promising one in Goddard's Visible Nulling Coronagraph (VNC).
\end{abstract}

Keywords: coronagraphy, interferometry, achromatic phase shift

\section{INTRODUCTION}

The direct observation of Terrestrial planets in the visible bandwidth would allow for spectroscopic analysis to determine planetary composition, as well as the presence of water and the possibility to support life. To achieve direct detection, an instrument must be capable of high-contrast imaging, or differentiating the 10 orders-of-magnitude difference between the host star's diffracted light and that reflected by an orbiting Terrestrial planet. Furthermore, as more and more telescopes are being designed with segmented or sparse apertures, the direct-detection technique should be compatible with these architectures ${ }^{1,2}$.

The visible nulling coronagraph (VNC) is a direct detection technique that achieves all of these requirements. As shown in Figure 1, the VNC uses a symmetric Mach-Zehnder interferometer to suppress the starlight at an inner working angle (IWA) of $\sim 2 \lambda / \mathrm{D}$. A MEMS segmented deformable mirror (DM) in one arm of the interferometer provides wavefront control. When coupled with a fiber-bundle array, simultaneous amplitude and phase control is achieved with a single DM. Furthermore, the fiber-bundle array spatially filters the wavefront, relaxing high spatialfrequency wavefront requirements on the system. The segmented nature of the DM also makes the VNC compatible with segmented and sparse aperture systems.

As the name implies, the VNC operates in the visible bandwidth regime. In order to enable spectroscopy of the detected planets, the VNC must also accommodate broad bandwidths, ideally spanning the band from $400 \mathrm{~nm}$ to $700 \mathrm{~nm}$. This implies that an achromatic phase shift of $\pi$ radians must be introduced between the arms of the interferometer. Many techniques for achieving an achromatic phase shift have been developed for white-light interferometry. For example, a series of dispersive plates can be designed to balance the optical path length (OPL) at several wavelengths (similar to the design of achromatic doublet lenses) ${ }^{3,4,5}$. Passing a beam through focus ${ }^{4,6}$, or sequential reflections from mirrored surfaces can also introduce a phase shift, at the cost of remapping the pupil of the optical system. Thin-film coatings can be used to achieve broadband performance of optics? ${ }^{7}$. Recently, a series of Fresnel rhomb prisms, augmented by sub-wavelength gratings, have been used to achieve an achromatic phase shift in the infrared ${ }^{8}$.

matthew.bolcar@nasa.gov 


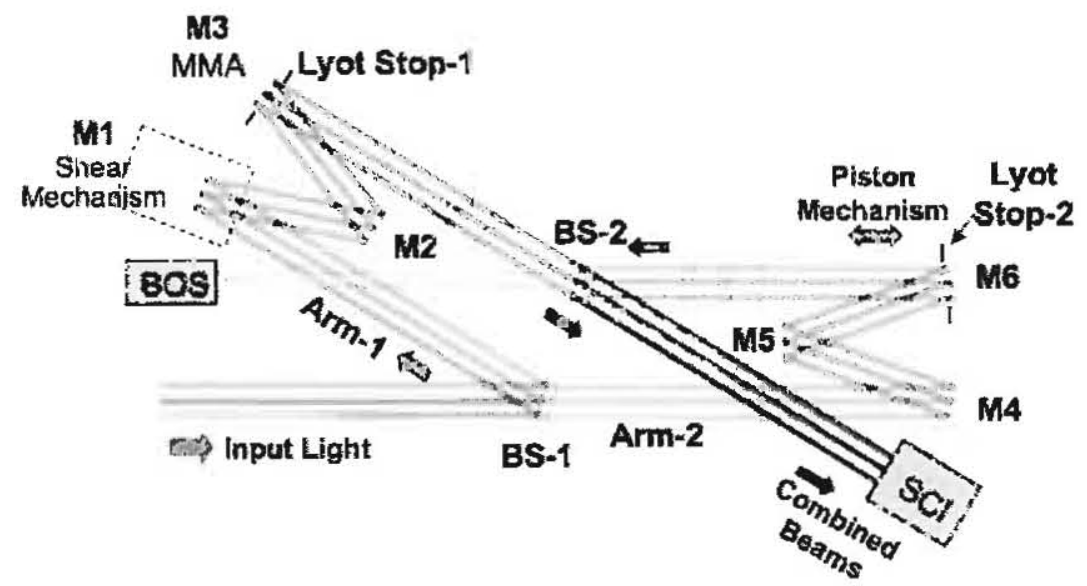

Figure 1-Optical layout of the visible nulling coronagraph. Light enters from the telescope at the lower left and is split and recombined by two matched beamsplitters. Light reflecting off the $1^{\text {st }}$ beamsplitter traverses two flats (M1 and M2) and reflects off a MEMS hex-packed segmented deformable mirror. This light both reflects and transmits through the $2^{\text {nd }}$ beamsplitter and is combined with the light reflecting off flats M4, M5 and M6. There are two output channels labeled as the bright object sensor (BOS) used for fine pointing and wavefront control, and the science (SCI) channel, where an in-focus image of star system without the starlight is collected.

While all of these techniques have been developed previously, none have to date achieved the extreme performance requirements for high-contrast imaging at broadband, visible wavelengths. We report here recent successes in two separate designs to achieve a broadband visible achromatic $\pi$-phase shift for the VNC.

\section{REQUIREMENTS OF ACHROMATIC PHASE SHIFTER}

\subsection{Contrast Dependence on Wavefront Error}

The achievable contrast of the VNC depends on a number of instrumental properties and ultimately determines several performance requirements on the achromatic phase shifter (APS). Before discussing those requirements, however, it is illustrative to discuss the dependence of contrast on something more straightforward: the difference in the wavefront error between the arms of the interferometer (for a more detailed discussion of the operation of the VNC, the reader is directed to references 1 and 9 ).

The noise-free image irradiances in the bright and dark focal planes of the VNC are given by

$$
\begin{aligned}
& I_{B}(\theta)=\frac{1}{2} I_{1} P S F_{0}(\theta)+\frac{1}{2} P S F_{\phi}(\theta)+\sqrt{I_{1} I_{2}} \operatorname{Re}\left\{A S F_{0}(\theta) A S F_{\phi}^{*}(\theta)\right\} \\
& I_{D}(\theta)=\frac{1}{2} I_{1} P S F_{0}(\theta)+\frac{1}{2} P S F_{\phi}(\theta)-\sqrt{I_{1} I_{2}} \operatorname{Re}\left\{A S F_{0}(\theta) A S F_{\phi}^{*}(\theta)\right\}
\end{aligned},
$$

where $I_{B}(\theta)$ and $I_{D}(\theta)$ represent the bright and dark channel output images, respectively, $\theta$ is the angular variable representing the focal plane projected on the sky, $I_{1}$ and $I_{2}$ are the integrated intensities in each arm of the interferometer such that $I_{1}+I_{2}=I_{\text {star }}, A S F_{0}(\theta)$ is the unaberrated, diffraction-limited complex amplitude spread function, $A S F_{\phi}$ is the aberrated (phase \& amplitude) complex amplitude spread function and $P S F_{0}$ and $P S F_{\phi}$ are the point spread functions given by

$$
\begin{aligned}
& P S F_{0}(\theta)=A S F_{0}(\theta) A S F_{0}^{*}(\theta) \\
& P S F_{\phi}(\theta)=A S F_{\phi}(\theta) A S F_{\phi}^{*}(\theta)
\end{aligned} .
$$


All of the phase and amplitude aberrations can be ascribed to one arm or the other of the interferometer without loss of generality since it is only the difference between the arms that matters.

Using the small angle approximation for the phase error of $e^{i \phi} \approx 1+i \phi$, we can expand the dark channel equation for $I_{D}(\theta)$, which leads to the following expression for image-plane contrast:

$$
C=\frac{I_{D}}{I_{\text {star }}} \approx\left(\frac{\pi W_{0}}{\lambda}\right)^{2} P S F_{0}\left(\theta-\theta_{0}\right),
$$

where $\theta_{0}$ is the location of the planet and $W_{0}$ is the amplitude of the wavefront error at the spatial frequency that corresponds to that location in the image plane. If the mean wavefront error is zero (equivalent to the piston difference between the interferometer arms being zero) then the average contrast is

$$
\langle C\rangle \approx\left(\frac{\pi W_{0}}{\lambda}\right)^{2}
$$

The brightness of speckles is exponentially distributed such that its mean is equal to the standard deviation of its intensity. Since we desire to set the requirements for the VNC based on high-confidence statistics we require

$$
\langle C\rangle+3 \sigma_{\langle c\rangle}=4\langle C\rangle \leq C_{\text {Flight }}
$$

where $C_{\text {Flight }}$ is the flight requirement contrast limit. This insures that the flight contrast limit will be met better than $99 \%$ of the time. Solving Eq. (4) for the wavefront error and computing the RMS value gives

$$
\sigma_{W} \approx \frac{1}{\sqrt{2}} \frac{\lambda}{\pi} \sqrt{\langle C\rangle}
$$

per spatial frequency.

For the lab VNC operating at a contrast of $C=10^{-8}$ at $\lambda=633 \mathrm{~nm}$ requires $\sigma_{\mathrm{W}} \approx 0.014 \mathrm{~nm}$ per spatial frequency. The overall RMS wavefront error (WFE) is obtained by integrating $\sigma_{W}$ over all spatial frequencies of interest. Spatial frequencies of interest are limited for the VNC to what is controllable by the segmented deformable mirror. The deformable mirror has 163 active segments, each with 3 control degrees of freedom (DOF), for a total of 489 control DOF in all. To achieve contrast of $10^{-8}$ with $489 \mathrm{DOF}$ at $\lambda=633 \mathrm{~nm}$ requires $\sigma_{W} \leq 0.247 \mathrm{~nm}$. This is the requirement on the RMS difference of the wavefront error between the two arms of the interferometer, if all other error sources are considered negligible.

\subsection{Contrast Dependence on the APS}

Of course, there are other error sources that are non-negligible, including intensity variations due to coating imperfections, polarization variations and errors due to a finite spectral bandpass. For each of these terms, an analysis similar to the one performed in Section 2.1 will yield requirements on how well the APS must control these error sources. We can re-express the bright- and dark-channel intensities from Eq. (1) in terms of the variance of each of the error terms:

$$
\begin{aligned}
& I_{B}(\theta)=\frac{1}{1+\frac{\pi^{4}}{8}|\theta|^{3}}\left\{1-\left[\pi^{2}\left(\frac{\sigma_{W}}{\lambda}\right)^{2}+\frac{\sigma_{I}^{2}}{16}+\frac{\sigma_{\psi}^{2}}{4}+\frac{\pi^{2}}{48}\left(\frac{\Delta \lambda}{\lambda_{0}}\right)^{2}\right]\right\} \\
& I_{D}(\theta)=\frac{1}{1+\frac{\pi^{4}}{8}|\theta|^{3}}\left[\pi^{2}\left(\frac{\sigma_{W}}{\lambda}\right)^{2}+\frac{\sigma_{I}^{2}}{16}+\frac{\sigma_{\psi}^{2}}{4}+\frac{\pi^{2}}{48}\left(\frac{\Delta \lambda}{\lambda_{0}}\right)^{2}\right]
\end{aligned}
$$


where $\sigma_{W}$ is the RMS wavefront error as computed in Section 2.1, $\sigma_{I}^{2}$ is the variance of the fractional intensity difference between the arms of the interferometer, $\sigma_{\psi}{ }^{2}$ is the variance of the difference in polarization-vector rotation between the arms in units of radians, $\Delta \lambda$ is the spectral bandwidth of the system, and we've made use of the asymptotic form of the PSF:

$$
\operatorname{PSF}(\theta)=\left[1+\frac{\pi^{4}}{8}|\theta|^{3}\right]^{-1}
$$

The last term in each expression in Eq. (7) is due to spectral leakage from the path length difference between the two arms of the interferometer. Ideally, the path length difference is half of the central wavelength, $\lambda_{0}$, to yield a phase difference of $\left(2 \pi / \lambda_{0}\right) O P L=\pi$ where $O P L$ is the optical path length difference. At wavelengths different than $\lambda_{0}$, the phase difference does not result in perfect destructive interference and light is leaked through, lowering the contrast. The primary role of the APS is to create an OPL such that the phase difference is constant as a function of the wavelength, effectively setting the fourth term of the expressions in Eq. (7) to zero.

Since some techniques for the APS employ a refractive element, it can introduce lateral chromatic color that is different in each arm of the VNC. Beams that shift differently as a function of wavelength in each arm create a mismatch in the output pupils, i.e. a shear. A Lyot stop masks out the edge of the pupil where the beams don't overlap, however it does interfere one beam against a slightly different part of the other beam. This yields a wavelengthdependent shear that leaks light through and lowers the contrast.

Assuming the electric field of the leaked light is given by

$$
E=\frac{1}{2}\left[e^{i \phi(x)}-e^{i \phi(x-\Delta s)}\right]
$$

where $\phi(x)$ and $\phi(x-\Delta s)$ are the phase error and the shifted phase error, respectively, then the focal-plane leakage term has the form

$$
I=P S F_{\phi} \sin ^{2}\left(\pi \frac{\Delta s}{D} \theta\right) \approx \frac{1}{1+\frac{\pi^{4}}{8}|\theta|^{3}}\left(\pi \frac{\Delta s}{D} \theta\right)^{2}
$$

where $\Delta s / D$ is the fractional beam shift due to the differential dispersion. Adding this term to the sum of error terms in Eq. (7) gives

$$
\begin{aligned}
& I_{B}(\theta)=\frac{1}{1+\frac{\pi^{4}}{8}|\theta|^{3}}\left\{1-\left[\pi^{2}\left(\frac{\sigma_{W}}{\lambda}\right)^{2}+\frac{\sigma_{I}^{2}}{16}+\frac{\sigma_{\psi}^{2}}{4}+\frac{\pi^{2}}{48}\left(\frac{\Delta \lambda}{\lambda_{0}}\right)^{2}+\left(\pi \frac{\Delta s}{D} \theta\right)^{2}\right]\right\} \\
& I_{D}(\theta)=\frac{1}{1+\frac{\pi^{4}}{8}|\theta|^{3}}\left[\pi^{2}\left(\frac{\sigma_{W}}{\lambda}\right)^{2}+\frac{\sigma_{I}^{2}}{16}+\frac{\sigma_{\psi}^{2}}{4}+\frac{\pi^{2}}{48}\left(\frac{\Delta \lambda}{\lambda_{0}}\right)^{2}+\left(\pi \frac{\Delta s}{D} \theta\right)^{2}\right]
\end{aligned}
$$

Using Eq. (11), along with the requirement that the contrast in Eq. (3) be $10^{-8}$, performance requirements of the APS with respect to each of the error terms can be generated. These performance requirements are summarized in Table 1 assuming $D=1 \mathrm{~cm}, \lambda=633 \mathrm{~nm}$ and $\theta=2 \lambda / D$. 
Table 1 - Performance Requirements of the APS

\begin{tabular}{|l|l|l|}
\hline Requirement & Value & Units \\
\hline RMS Phase Shift & $<0.00045$ & Radians \\
\hline RMS Intensity Difference & $<0.0005$ & Percent \\
\hline RMS Polarization Rotation Difference & $<0.00017$ & Radians \\
\hline Differential Dispersion Beam Shift & $<2.0$ & Microns \\
\hline
\end{tabular}

\section{GLASS PLATE APS}

\subsection{Theory}

The theory behind the dispersive element achromatic phase shifter is closely related to the design of achromatic lenses. By balancing the wavelength-dependent indexes of refraction of different materials, the optical path length through the element can be made almost uniform for multiple wavelengths. Generally speaking, more dispersive elements are required to correct a broader range of wavelengths. For example, an achromatic lens is usually a doublet that corrects the optical path at two design wavelengths, while an apochromatic lens is usually a triplet and corrects the optical path for three design wavelengths.
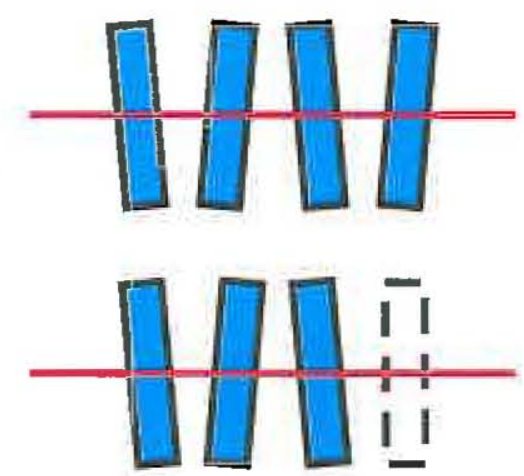

Figure 2 - Example arrangement of glass plates. Each plate is tilted by $2.5^{\circ}$ to prevent ghost reflections from propagating through the system. The dashed outline indicates that one arm of the interferometer may include an additional airspace in order to achieve the achromatic phase shift.

For the VNC, the dispersive elements have no optical power, which is to say they are simple glass plates with parallel surfaces. However, to prevent ghost reflections off each of the plate facets from propagating through the system, the windows are oriented at an angle to each other of approximately $2.5^{\circ}$. Figure 2 shows an example layout for the glass plates. For such an arrangement, the optical path length through the plates is given by

$$
O P L(\lambda)=\sum_{j=1}^{N_{\text {patak }}} \frac{t_{j} n_{j}(\lambda)}{\cos \alpha_{j}^{\prime}(\lambda)},
$$

where $t_{j}$ is the thickness of the $j^{\text {th }}$ plate, $\alpha_{j}^{\prime}(\lambda)$ is the angle of refraction at the $j^{\text {th }}$ plate and $n_{j}(\lambda)$ is the index of refraction of the $j^{\text {th }}$ plate. The phase shift between the arms of the interferometer is then given by

$$
\Delta \Phi(\lambda)=\frac{2 \pi}{\lambda}\left[O P L(\lambda)_{a r m 1}-O P L(\lambda)_{a r m 2}\right]
$$

Arranging the plates in an angled configuration, however, introduces several other effects on the optical beam that must be mitigated. At non-normal incidence, the beam will refract as it enters each glass plate, and again as it exits. After passing through several glass plates, the beam will have dispersed laterally in one dimension as a function of 
wavelength, as demonstrated in Figure 3. The beam displacement when passing through a series of glass plates at nonnormal incidence is give by

$$
D(\lambda)=\sum_{j=1}^{N_{\text {Rlate }}} t_{j}\left[1-\frac{\cos \alpha_{j}}{n_{j}(\lambda) \cos \alpha_{j}^{\prime}(\lambda)}\right]
$$

where $\alpha_{j}$ is the angle of incidence at the $j^{\text {th }}$ plate. As discussed in Section 2, the differential dispersed beam width between the arms of the interferometer, $\Delta D=\left|D(\lambda)_{\text {arm } 1}-D(\lambda)_{\text {arm } 2}\right|$, must be less than $2 \mu \mathrm{m}$.

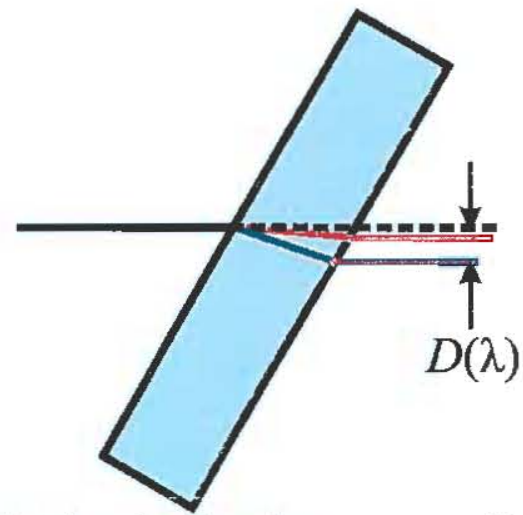

Figure 3 - Beam displacement when passing through a glass plate at non-normal incidence. The total difference in the beam displacement between the two arms must be small enough that when re-combined, the two beams interfere with the same regions.

Also, it is well known that a linearly polarized beam passing through a plate at non-normal incidence will undergo a slight rotation of its polarization angle relative to plane of incidence ${ }^{10}$. If $P_{i}(\lambda)$ is the initial angle between the plane of polarization and the plane of incidence, then after transmitting through the glass plates, the angle will be

$$
P_{o}(\lambda)=P_{i}(\lambda) \prod_{j=1}^{N_{\text {ploses }}} \cos \left[\alpha_{j}-\alpha_{j}^{\prime}(\lambda)\right] \cos \left[\alpha^{\prime}(\lambda)_{j}-\alpha_{j}\right]
$$

assuming a small-angle approximation, and where the first cosine term corresponds to the beam entering a particular glass plate and the second cosine term corresponds to the beam exiting that same plate. The standard deviation of the differential change in polarization between the arms of the interferometer, $\Delta P_{o}(\lambda)=\left|P_{o}(\lambda)_{\text {arm } 1}-P_{o}(\lambda)_{\text {arm } 2}\right|$, must be less than 0.00017 radians.

Finally, at each air-glass interface, the beam intensity will be reduced by Fresnel reflection and transmission losses. Through a single arm of the interferometer, the Fresnel intensity transmission coefficient is given by

$$
T(\lambda)=\prod_{j=1}^{N_{\text {plawe }}}\left\{1-\frac{\sin ^{2}\left[\alpha_{j}(\lambda)-\alpha_{j}^{\prime}(\lambda)\right]}{\sin ^{2}\left[\alpha_{j}(\lambda)+\alpha_{j}^{\prime}(\lambda)\right]}\right\}\left\{1-\frac{\sin ^{2}\left[\alpha_{j}^{\prime}(\lambda)-\alpha_{j}(\lambda)\right]}{\sin ^{2}\left[\alpha_{j}^{\prime}(\lambda)+\alpha_{j}(\lambda)\right]}\right\},
$$

where the first term in braces corresponds to the beam entering a particular glass plate and the second term in braces corresponds to the beam exiting the same plate. The standard deviation of the differential change in intensity between the arms of the interferometer, $\Delta T(\lambda)=\left|T(\lambda)_{a r m 1}-T(\lambda)_{\text {arm2 }}\right|$, must be less than $0.0005 \%$.

\subsection{Design Procedure}

The design of the glass-plate APS is one of optimization: what selection of plate materials and thicknesses minimizes the differential lateral dispersion, transmitted intensity and change in polarization, while achieving the achromatic phase shift? The optimization procedure is complicated by the fact that one set of variables, the plate materials, is discrete 
while the other set, plate thicknesses, is continuous. We used an in-house global search routine that generates configurations by selecting glass materials from a database. These configurations are optimized by following several trajectories through the search-space using a recursive branching structure. For each configuration that is tested, the glass plate thicknesses are optimized by a constrained nonlinear optimization routine. We found this in-house routine to be significantly more efficient than a traditional global search algorithm such as simulated annealing.

The error metric that is evaluated by the optimization routine is a weighted sum of components for each of the conditions presented in Section 3.1. The error metric components for the phase shift, transmitted intensity difference, and differential change in polarization are the mean-squared-errors, given by

$$
\begin{gathered}
E_{O P L}=\sum_{\lambda}[\Delta \Phi(\lambda)-\pi]^{2}, \\
E_{T}=\sum_{\lambda}\left[T(\lambda)_{a m m}-T(\lambda)_{a r m 2}\right]^{2},
\end{gathered}
$$

and

$$
E_{P}=\sum_{\lambda}\left[P_{o}(\lambda)_{a r m 1}-P_{o}(\lambda)_{a r m 2}\right]^{2}
$$

The error metric component for the lateral dispersion component is the standard deviation of the differential lateral dispersion, since mean errors can be removed through re-alignment of the two arms of the interferometer. The error metric component is given by

$$
E_{D}=\sqrt{\frac{1}{N_{\lambda}} \sum_{\lambda}[\Delta D(\lambda)-\overline{\Delta D(\lambda)}]^{2}},
$$

where the overbar denotes computing the mean value of the quantity.

The total error metric to be minimized by the global search routine is a weighted sum of these components:

$$
E=\gamma_{1} E_{O P L}+\gamma_{2} E_{D}+\gamma_{3} E_{T}+\gamma_{4} E_{P}
$$

where the relative weights allow us to emphasize certain components over the others, if needed. Analytic derivatives of $E$ with respect to the glass plate thicknesses are easily computed to facilitate the nonlinear optimization portion of the search.

\subsection{Design Results}

Initially, a large glass database was used to generate the random configurations used in the global search algorithm, and several solutions were found. However, to improve manufacturability of the glass plates, a second round of optimizations was performed with a restricted, "preferred" database of commonly used glasses that are easy to work with. Table 2 shows the location, material and thickness of each plate. 
Table 2 - Glass Plate Achromatic Phase Shifter Solution

\begin{tabular}{|l|l|l|}
\hline Location & Material & Thickness $\mathbf{( m m}$ ) \\
\hline Arm 1 & Ohara S-TIM25 & 3.835 \\
\hline Arm 1 & Ohara S-BSM28 & 5.199 \\
\hline Arm 1 & Ohara S-TIH4 & 9.395 \\
\hline Arm 2 & Ohara S-BSM28 & 3.519 \\
\hline Arm 2 & Ohara S-THH1 & 14.780 \\
\hline Arm 2 & Vacuum & 0.240 \\
\hline
\end{tabular}

This solution uses five individual plates and four distinct materials and provides an achromatic phase shift over the bandwidth between $530 \mathrm{~nm}$ and $730 \mathrm{~nm}$. Figure 4 - Figure 7 show the phase shift error, differential lateral dispersion, differential transmitted intensity, and differential change in polarization, respectively, as functions of wavelength. Table 3 summarizes the performance metrics of the solution.

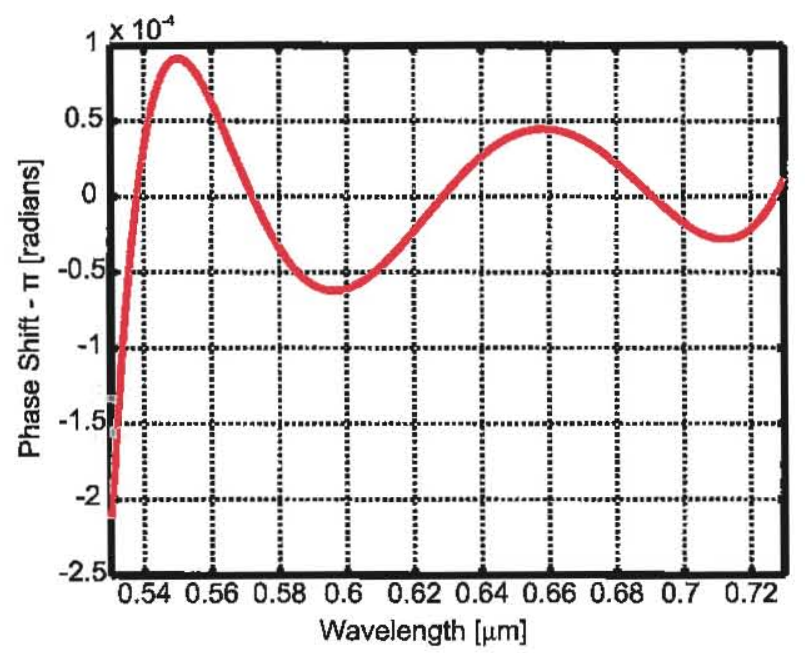

Figure $4-$ The error in the phase shift, $\left[\Phi_{\text {arml }}(\lambda)-\Phi_{\text {arm2 }}(\lambda)\right]-\pi$. The mean error is $-1.44 \times 10^{-9}$ radians with a standard deviation of $4.70 \times 10^{-5}$ radians.

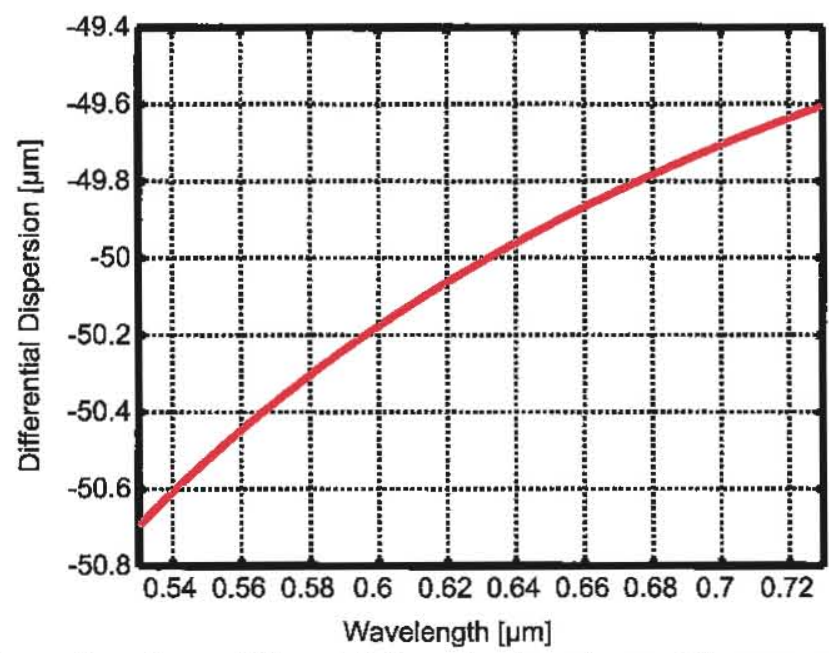

Figure 5 - The beam shear due to differential dispersion in each arm. The mean error is $-50.06 \mu \mathrm{m}$, which can be removed through re-alignment. The standard deviation of the error is $0.31 \mu \mathrm{m}$. 


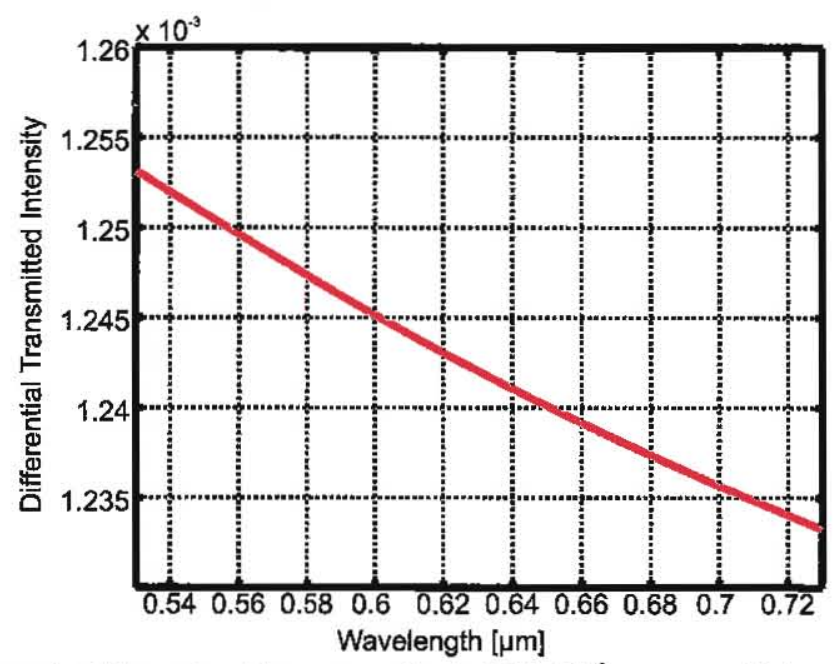

Figure 6 - The differential transmitted intensity. The mean value is $1.24 \times 10^{-3}$ percent, which can be removed by balancing the average intensity in each arm of the interferometer. The standard deviation is $5.78 \times 10^{-6}$ percent.

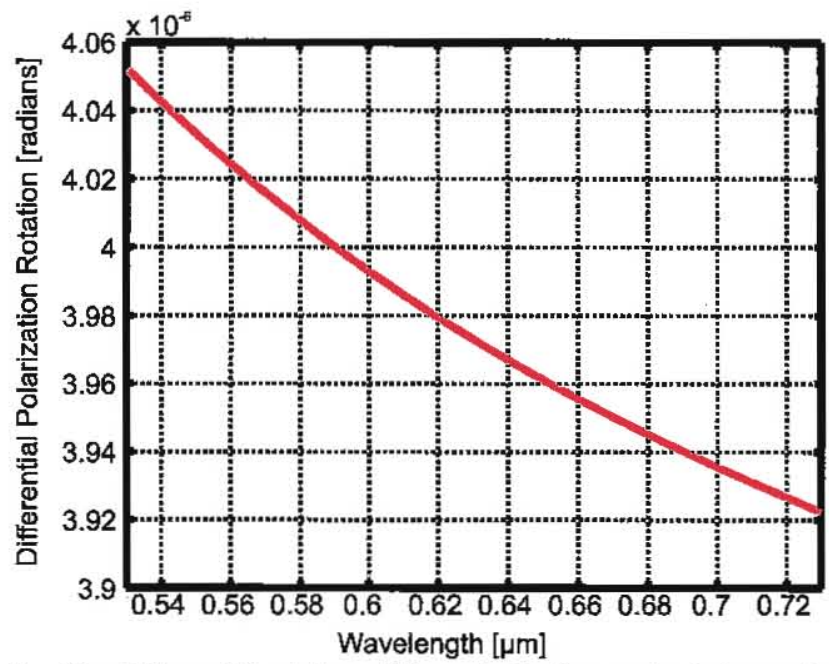

Figure 7 - The differential rotation of the polarization vector between the arms.

The mean value is $3.98 \times 10^{-6}$ radians and the standard deviation is $3.72 \times 10^{-8}$ radians.

Table 3 - Performance results for the glass-plate APS solution presented in Table 2.

\begin{tabular}{|l|l|l|}
\hline Quantity & Mean Error & Standard Deviation \\
\hline Phase Shift & $-1.44 \times 10^{-9}$ radians & $4.70 \times 10^{-5}$ radians \\
\hline Beam Shear & $-50.06 \mu \mathrm{m}$ & $0.31 \mu \mathrm{m}$ \\
\hline Differential Intensity & $1.24 \times 10^{-3} \%$ & $5.78 \times 10^{-6} \%$ \\
\hline $\begin{array}{l}\text { Differential Rotation of the } \\
\text { Polarization Vector }\end{array}$ & $3.98 \times 10^{-6}$ radians & $3.72 \times 10^{-8}$ radians \\
\hline
\end{tabular}

\subsection{Manufacturability \& Alignment}

While this solution meets all of the necessary performance requirements, it proves to be infeasible from a manufacturing and alignment perspective. Material imperfections play a big role in terms of striae, or non-uniformities in the index of refraction of the glass. It is possible to obtain high-quality melts of some glass materials that would meet the stringent zero-tolerance of the VNC APS. However, for the glasses listed in Table 2, such quality is not guaranteed.

Polishing the glass plates poses another challenge. The thickness of the glass plate is the dominant contributor to the OPL through the plate, and must be controlled to better than $\pm 25 \mu \mathrm{m}$. While it is possible to achieve such 
accuracy with modern polishing techniques, it forces other tolerances such as surface flatness, surface roughness, and surface quality (i.e. scratch-dig) to be relaxed. Ultimately, the polishing of each plate is a best-effort attempt, with no guarantee that the requirements will be met. It is possible that some error in the plate thickness can be compensated by adjustments in the angle of the glass plate. Preliminary tolerancing of the alignment of the plates, however, shows that the angular alignment needs to be achieved with arc-second accuracy in the lab.

\section{THIN-FILM FRESNEL RHOMB APS}

\subsection{Theory}

A second technique of achieving the APS uses a pair of Fresnel rhomb prisms in each arm of the interferometer and was first reported by Mawet, et. al., for use in the infrared regime ${ }^{8}$. The concept leverages the polarization-dependent phase shift that occurs when a beam undergoes total internal reflection (TIR). A schematic of the device is shown in Figure 8. The phase shift between the $s$ and $p$ polarization states for a single TIR is given by

$$
\Delta \Phi_{s-p}(\lambda)=2 \arctan \left[\frac{\sqrt{\sin ^{2} \alpha-n_{t e}^{2}(\lambda)}}{n_{t e}^{2}(\lambda) \cos \alpha}\right]-2 \arctan \left[\frac{\sqrt{\sin ^{2} \alpha-n_{t e}^{2}(\lambda)}}{\cos \alpha}\right]
$$

where $\alpha$ is the angle of incidence at the interface, and $n_{t i}(\lambda)$ is the ratio of the emergent and incident indexes of refraction:

$$
n_{t i}(\lambda)=\frac{n_{\text {emergent }}(\lambda)}{n_{\text {incident }}(\lambda)}
$$

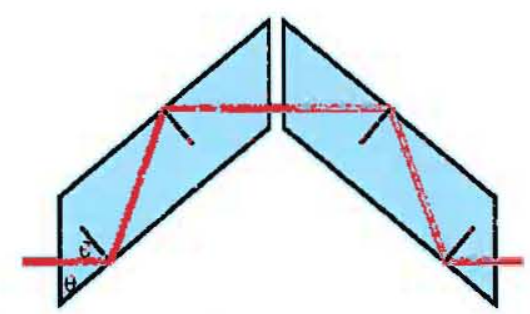

Figure 8-A diagram of the Fresnel rhomb APS. The beam undergoes 4 total-internal reflections (TIR). At each TIR, a polarization-dependent retardance is introduced in the beam. The acute prism angle determines the angle of incidence at the TIR interface.

The phase shift as a function of angle-of-incidence is shown in Figure 9 for TIR at a fused-silica/air interface for several wavelengths. There are two aspects to note: First, the phase shift is nearly achromatic for this selection of materials, though not enough to meet the VNC's requirements. Second, the maximum phase shift achieved at an angle of incidence of $53^{\circ}$ is only 0.74 radians. Even after the four TIR bounces in the device shown in Figure 8, the total phase shift is still less than $\pi$.

Mawet, et. al., used a subwavelength grating at the TIR interfaces to augment the phase shift in the infrared, achieving not only the necessary phase shift magnitude, but also achromaticity. They also explored the use of thin-film coatings at the TIR interfaces - a technique we optimize here for visible wavelengths. If $r$ is the amplitude reflection coefficient of the thin-film coating, then the phase of the reflected beam is $\arctan (r)$. The polarization-dependent phase shift is then given by

$$
\Delta \Phi_{s-p}(\lambda)=\arctan \left[r_{s}(\lambda)\right]-\arctan \left[r_{p}(\lambda)\right]
$$

where $r_{s}$ is the amplitude reflection coefficient for $s$-polarization and $r_{p}$ is the amplitude reflection coefficient for $p$ polarization. 


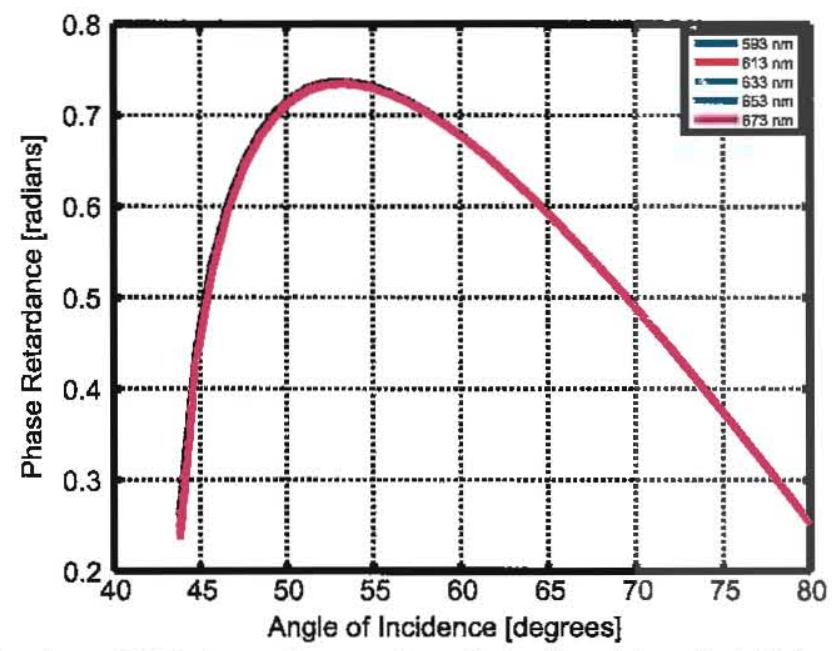

Figure 9 - The phase shift between the $s$ - and p-polarization states at total internal reflections.

The phase shift is nearly achromatic, and has a peak at $53^{\circ}$ of 0.74 radians.

Through proper selection of the substrate material, angle-of-incidence, coating layer thickness, coating layer material, and the number of coating layers, one can tune the polarization-dependent phase shift to be achromatic and equal to $\pi$. A second device in the opposite arm of the interferometer, rotated by $90^{\circ}$, introduces the phase shift in the opposite polarization state. For example, in the first arm of the interferometer, the $p$-polarization state is retarded by $\pi$ radians relative to the $s$-polarization state. Conversely, in the second arm of the interferometer, the $s$-polarization state is retarded by $\pi$ radians relative to the $p$-polarization state. Thus, when the beams are recombined, each polarization state destructively interferes to produce a null.

\subsection{Design Process}

The design of the Fresnel rhomb prisms was performed similarly to the glass-plate APS. A global search routine was run over the free-variables of angle-of-incidence, coating layer material, and coating layer thickness to achieve the requirements. The substrate was chosen to be Schott LITHOSIL, as high-quality melts with no striae or inclusions can be obtained. Initially, the coating was constrained to have only two layers for simplicity. The global search routine generated coating configurations by drawing materials from a pre-specified database, and then optimized the angle-ofincidence and each layer thickness by constrained nonlinear optimization.

Unlike the glass plate APS, the error metric that was optimized consists of only a single term related to the phase shift. This simplification is due to several reasons. First, in the nominal design of the APS, the beam does not undergo refraction anywhere, so there is no beam shear due to differential dispersion. Furthermore, since all reflections are TIR, the Fresnel rhombs provide $100 \%$ throughput in each arm (ignoring absorptive effects of the substrates, which should be equivalent in each arm anyway). Also due to each reflection being TIR, there is no rotation of the polarization vector at each interface; the polarization state of the beam is preserved.

The error metric minimized by the global search routine is just

$$
E=\sum_{\lambda} \Delta \Phi_{s}^{2}(\lambda)+\sum_{\lambda} \Delta \Phi_{p}^{2}(\lambda)
$$

where $\Delta \Phi_{s}$ and $\Delta \Phi_{p}$ are the phase differences between the arms of the interferometer for $s$ - and $p$-polarization, respectively.

\subsection{Design Results}

Several solutions were found that achieved the VNC's requirements; the one with the best performance was selected for fabrication. Figure 10 shows a schematic of a single prism, including the coating prescription for each surface. All four prisms are identical. Figure 11 shows the phase shift performance for each polarization state over the $40-\mathrm{nm}$ 
bandwidth centered about $632.8 \mathrm{~nm}$. Table 4 summarizes the design \& performance parameters of the Fresnel rhomb APS.

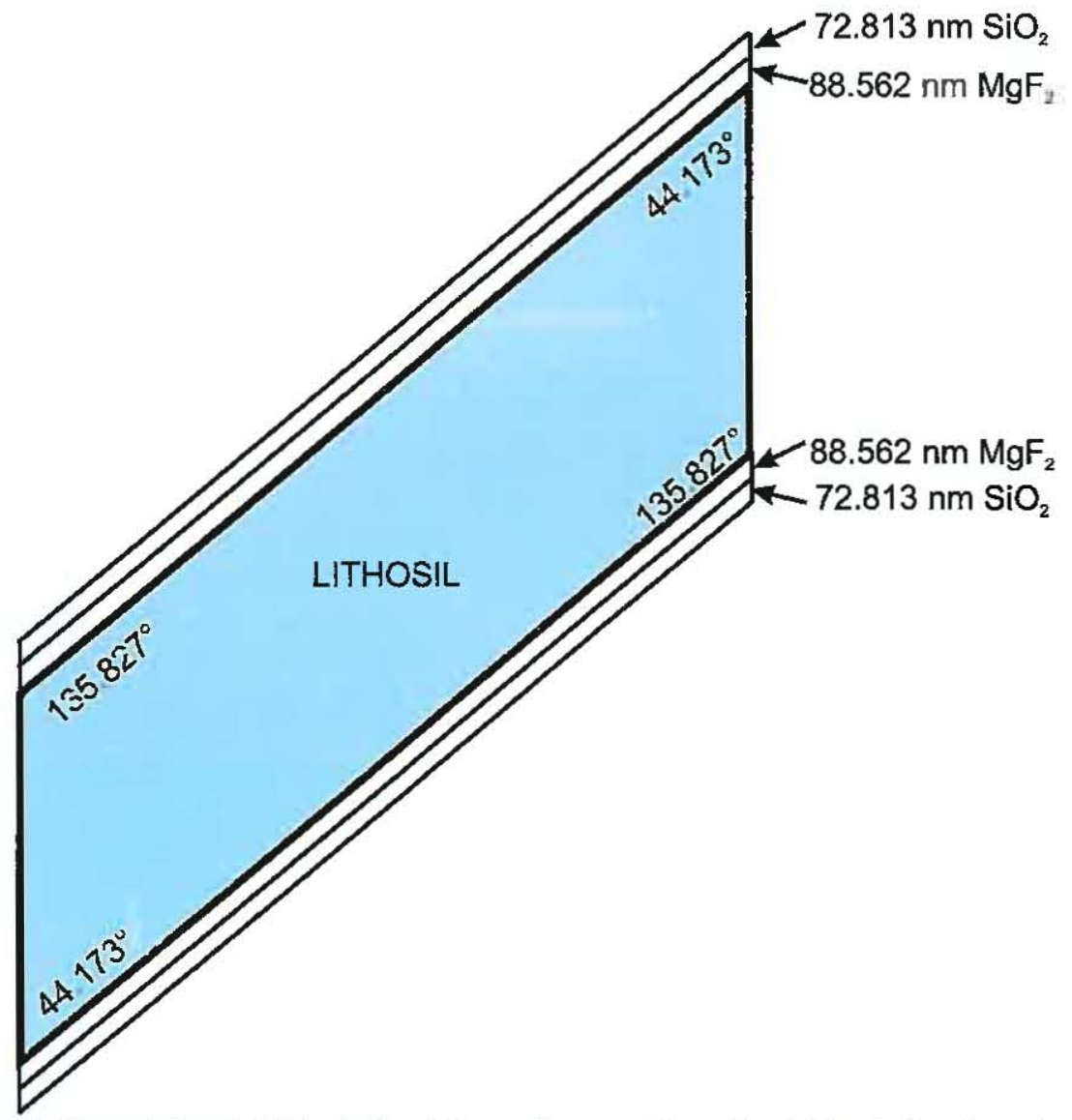

Figure 10 - The Fresnel rhomb APS solution. The coating on each surface is identical, and consists of just two layers. All four prisms are identical.

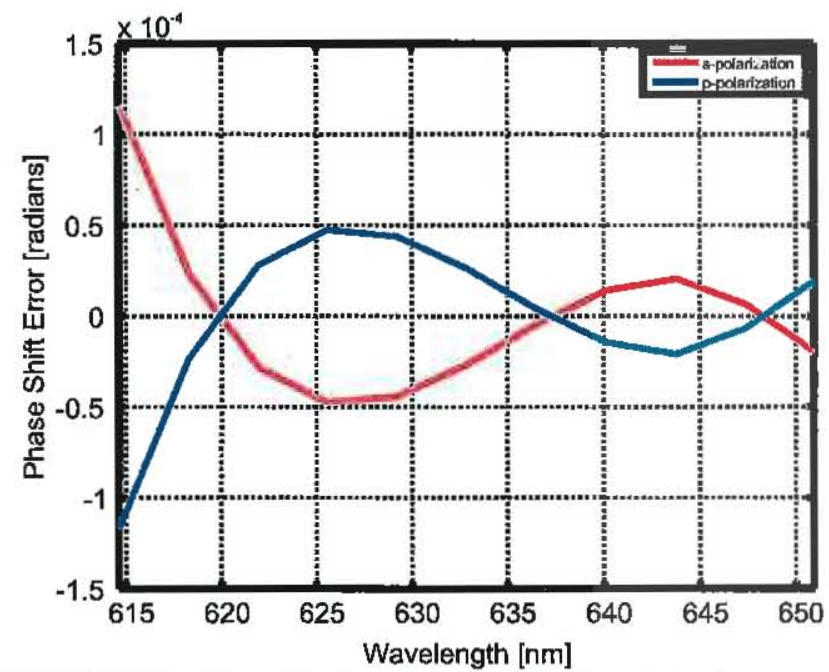

Figure 11 - The phase shift error, $\left[\Phi_{\text {arm } 1}(\lambda)-\Phi_{\text {arm2 }}(\lambda)\right]-\pi$, in each polarization state. The mean error for $\mathrm{s}$-polarization is $4.14 \times 10^{-7}$ radians with a standard deviation of $4.35 \times 10^{-5}$ radians. For p-polarization, the mean error is $-1.24 \times 10^{-6}$ radians with a standard deviation of $4.35 \times 10^{-5}$ radians. 
Table 4 - Fresnel rhomb prism APS design parameters and performance values.

\begin{tabular}{|l|l|}
\hline Parameter: & Value: \\
\hline Substrate Material & Schott LITHOSIL \\
\hline Prism Acute Angle & $44.173^{\circ}$ \\
\hline Prism Obtuse Angle & $135.827^{\circ}$ \\
\hline Coating Layer 1 Material & $\mathrm{MgF}_{2}$ \\
\hline Coating Layer 1 Thickness & $88.562 \mathrm{~nm}$ \\
\hline Coating Layer 2 Material & $\mathrm{SiO}_{2}$ \\
\hline Coating Layer 2 Thickness & $72.813 \mathrm{~nm}$ \\
\hline \multicolumn{2}{|l|}{} \\
\hline $\begin{array}{l}s \text {-Polarization Mean Phase- } \\
\text { shift Error }\end{array}$ & $4.14 \times 10^{-7}$ radians \\
\hline $\begin{array}{l}s \text {-Polarization Phase-shift } \\
\text { Error Standard Deviation }\end{array}$ & $4.35 \times 10^{-5}$ radians \\
\hline $\begin{array}{l}p \text {-Polarization Mean Phase- } \\
\text { shift Error }\end{array}$ & $-1.24 \times 10^{-6}$ radians \\
\hline $\begin{array}{l}p \text {-Polarization Phase-shift } \\
\text { Error Standard Deviation }\end{array}$ & $4.35 \times 10^{-5}$ radians \\
\hline
\end{tabular}

\subsection{Discussion}

From a manufacturing perspective, the Fresnel rhomb APS appears to be more feasible than the glass plate APS. Since all four prisms are identical in design, they can be polished as a single block and later diced, reducing the variation in polishing errors of the prism angle. The prisms can also be coated as a group, reducing variation in coating layer thickness.

Tolerances on both the prism angle and layer thicknesses are within standard manufacturing precision. The prism angle (which determines the angle-of-incidence for the TIR reflections) is actually a fairly loose tolerance. Once the prisms are manufactured, the prism angle can be measured to arc-second accuracy and the coating prescription can be re-optimized to accommodate. Coating layers can routinely be controlled during deposition with nanometer accuracy.

\subsection{Variations to the Design}

We are currently exploring a variation to the design of the Fresnel rhomb APS, as well as running a more detailed tolerancing analysis to determine the manufacturability of the device. The design variation is required to mitigate ghost reflections between the prism pairs in each arm. A wedge angle is added to the input and output facets of each prism, as shown in Figure 12. The biggest impact this has on performance is that it re-introduces beam shear due to differential dispersion since the beams now refract upon entering and exiting each prism. Furthermore, since the device in each arm is rotated by $90^{\circ}$ with respect to its counterpart, the beams shear in orthogonal dimensions. We are still exploring the impact of this on the APS performance.

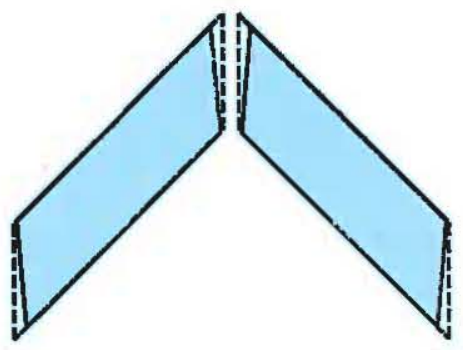

Figure 12 - Fresnel rhomb APS with wedged interfaces to direct ghost reflections out of the system.

A Monte Carlo study is also being performed on the manufacturing process to determine the potential yield of devices that will meet the VNC's requirements. Using the baseline design, typical polishing errors are added to the 
prism angle and the coating prescription is re-optimized to achieve the desired phase shift. Random manufacturing errors are then added to the coating layer thicknesses to determine the final performance of the device. The results of this study are not yet available.

\section{CONCLUSION}

We report here on two new designs to achieve an achromatic phase shift for the visible nulling coronagraph. The APS is required to achieve high-contrast, broadband direct detection of exoplanets. The first design uses a series of dispersive glass plates to achieve a $\pi$-phase shift across the band between $530 \mathrm{~nm}-730 \mathrm{~nm}$. The RMS error of the achieved phase shift is $4.7 \times 10^{-5}$ radians. Differential intensity, lateral beam dispersion, differential polarization rotation and ghost images are also minimized. Ultimately, the glass plate solution proved too difficult to manufacture and align in the lab VNC.

The second design uses Fresnel rhomb prisms and leverages the polarization-dependent retardance that occurs at total-internal reflection. Thin film coatings on the TIR interfaces are used to augment the retardance to achieve the desired phase shift as well as achromaticity. The optimized solution uses four LITHOSIL-substrate prisms with twolayer coatings of $\mathrm{SiO}_{2}$ on $\mathrm{MgF}_{2}$ to achieve the phase shift. Two prisms are used in each arm, with one arm rotated by $90^{\circ}$ to achieve the complementary retardance.

The Fresnel rhomb APS appears to be more easily manufactured and aligned than the glass plate APS. Further study is being performed to determine tolerances on the prism angles and coating prescription.

\section{REFERENCES}

[1] Lyon, R.G, Clampin, M., Woodruff, R.A., Vasudevan, G., Thompson, P., Chen, A., Petrone, P., Booth, A., Madison, T., Bolcar M.R., Noecker, M.C., Kendrick, S., Melnick, G., and Tolls, V., "Visible nulling coronagraphy testbed development for exoplanet detection", Proc. SPIE 7731, 77312B (2010).

[2] Lyon, R.G., Oegerle, W.R., Feinberg, L.D., Bolcar, M.R., Dean, B.H., Mosier, G.E., and Postman, M., "Coronagraphic wavefront control for the ATLAST 9.2m telescope", Proc. SPIE 7731, 77312S (2010).

[3] Ballard, P.T., Hill, H.A., and Figoski, J.W., "Broadband Optical Phase Shifter," Appl. Opt. 17, 331-333 (1978).

[4] Gappinger, R.O., Diaz, R.T., Ksendzov, A., Lawson, P.R., et al., "Experimental evaluation of achromatic phase shifters for mid-infrared starlight suppression," Appl. Opt. 47, 868-880 (2009).

[5] Mieremet, A.L., Braat, J.J.M., Bokhove, H., and Ravel, K., "Achromatic phase shifting using adjustable dispersive elements," Proc. SPIE 4006, 1035 (2000).

[6] Hénault, F., "Design of achromatic phase shifters for spaceborne nulling interferometry," Opt. Lett. 31, 3635-3637 (2006).

[7] Lemarquis, F., and Riaud, P., "Thin-film achromatic phase shifters for nulling interferometry: design approach," Appl. Opt. 42, 6919-6928 (2003).

[8] Mawet, D., Hanot, C., Lenaers, C., Riaud, P., et al., "Fresnel rhombs as achromatic phase shifters for infrared nulling interferometry," Opt. Express 15, 12850-12865 (2007).

[9] Lyon, R.G., Clampin, M.C., Petrone, P., Mallik, U., Madison, T.J., Bolcar, M.R., "High contrast vacuum nuller testbed (VNT) contrast, performance, and null control," Proc. SPIE 8442 (2012).

[10] Born, M., and Wolf, E., Principles of Optics, $7^{\text {th }}$ Ed., Cambridge University Press, Cambridge, U.K., pg. 47 (1999). 


\title{
Approaches for Achieving Broadband Achromatic Phase Shifts
} for Visible Nulling Coronagraphy

Matthew R. Bolcar and Richard G. Lyon

NASA Goddard Space Flight Center, 8800 Greenbelt Rd., Greenbelt, MD 20771

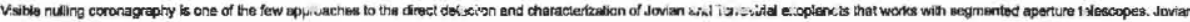

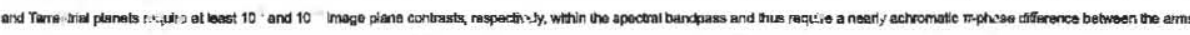

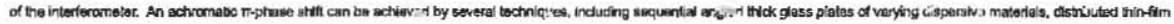

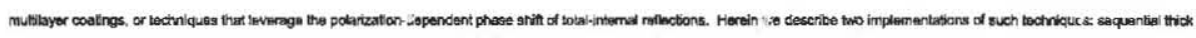

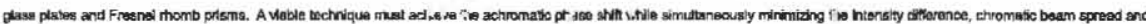

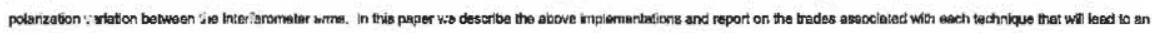

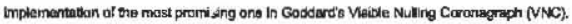

\section{Introduction}

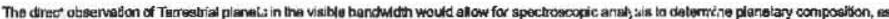

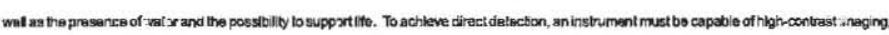

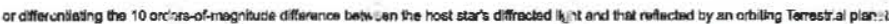

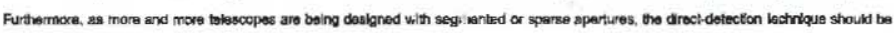

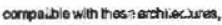

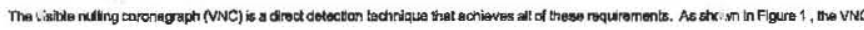

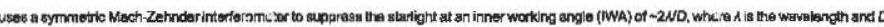

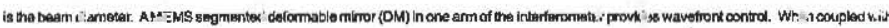

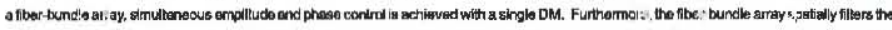

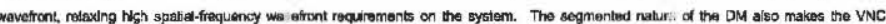

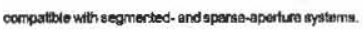

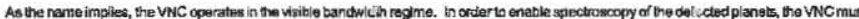

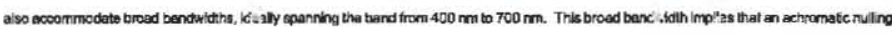

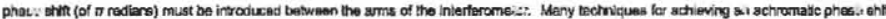

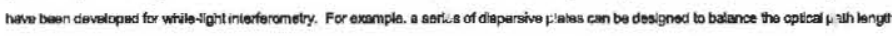

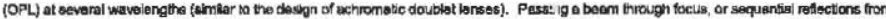

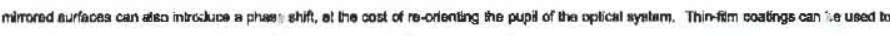

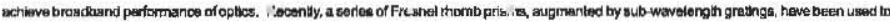
ochleve an schromatlc phase shiftin ithe infrared.
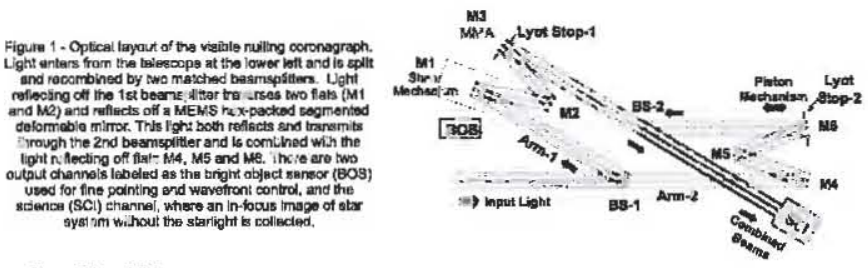

\section{Glass-PlateAPS}

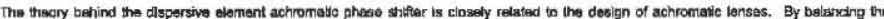

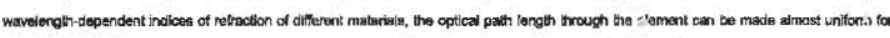

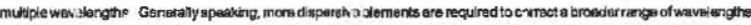

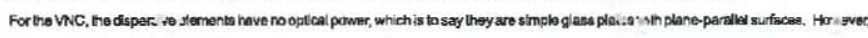

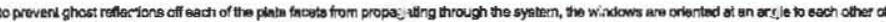

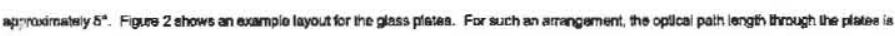
given by

$$
\operatorname{OP} L(\lambda)=\sum_{j=1}^{n} \cos \alpha_{i}^{n}(\lambda)
$$

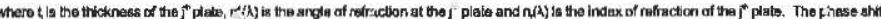
betwasn the arrms of the interfercreier is then given by

$$
\triangle \Phi(\lambda)=\frac{2 \pi}{\lambda}\left[O P L(\lambda)_{-\infty}-O P L(\lambda)=2\right]
$$

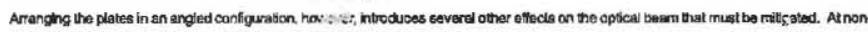

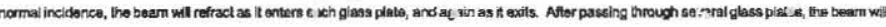

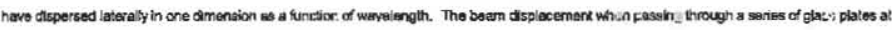
nom-nomatinaidance is given by

$$
\mathrm{a}(\lambda)=\sum_{j=1} \mathrm{t}_{\mathrm{i}}\left[1-\frac{\cos \alpha}{\mathrm{n}_{1}(\lambda) \cos \alpha_{j}(\lambda)}\right]
$$

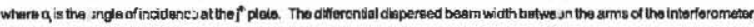

$\Delta B=\mid B(\lambda),-B(\lambda)$, nust be less than $2 \mu \mathrm{m}$

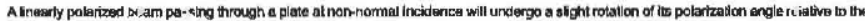

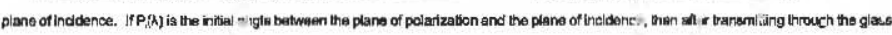
plestes, the anglis wh be

$$
P_{0}(\lambda)=P_{i}(2) \prod_{j}^{n} \cos \left[\alpha_{-}-\alpha_{f}^{\prime}(\lambda)\right] \cos \left[\alpha\left(\lambda_{1}-\alpha\right]\right.
$$

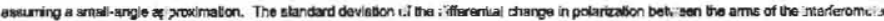

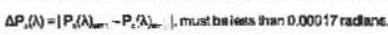

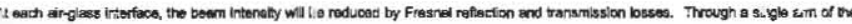

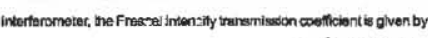

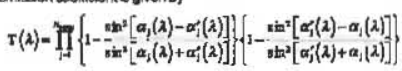

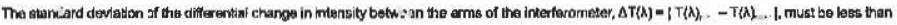
$0.0005 \%$

\section{Thin-Film Fresne! RhombAPS}

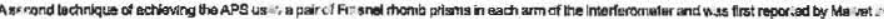

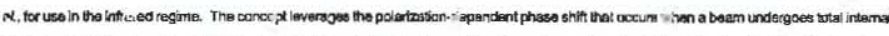

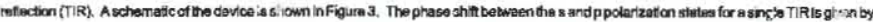

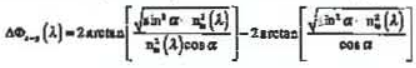

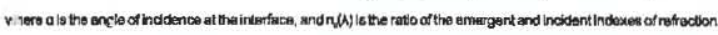

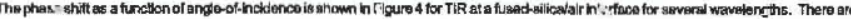

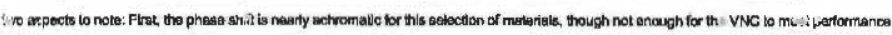

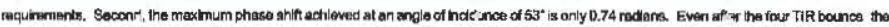
to is phose enhiftis sall less then n.

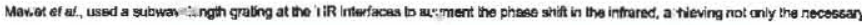

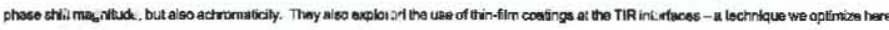

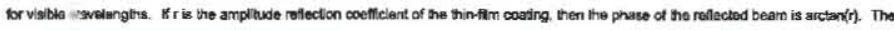
polarizatond dendent phas s shit is then given to

$$
\Delta \omega_{n \rightarrow}(\lambda)-\arctan [[z(\lambda)]-\sin \min [s(\lambda)]
$$

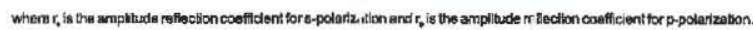
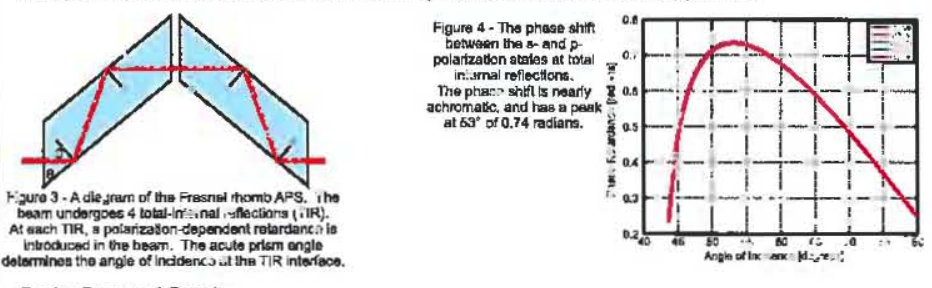

Design Process \& Results

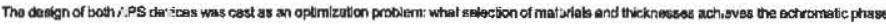

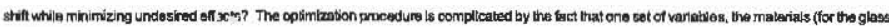

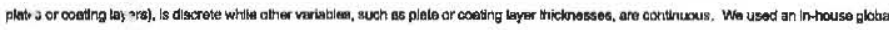

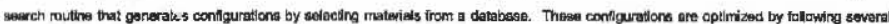

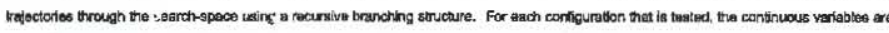

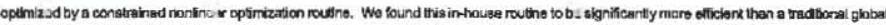

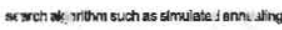

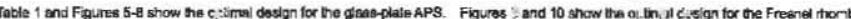
prismAPS.

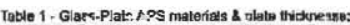

Locatton Materlal Thloknocs (mm)

Amm 1 Ohase S-TIM25 3.335

Am 1 Onara S-BSM28 5.190

Arm 1 Oharr S-TIH4 9.995

Amn 2 Onera SBSMm28 3.519

Amn 2 Ohara S-TIH1 14.780

ism 2 vacum 0.240
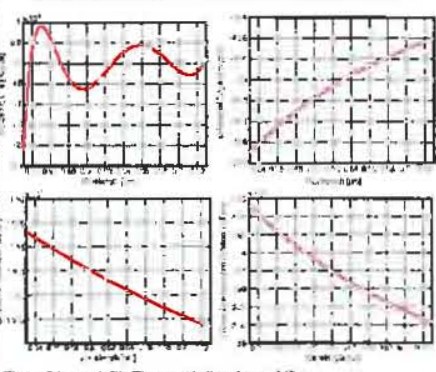

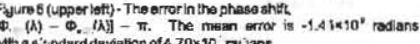

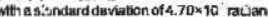

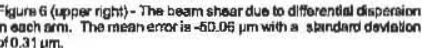

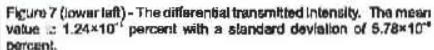

Four a A (lower righc) - The differcindal rotation of the polurization velor between the arms. The mean valus is $3.98 \times 10^{-4}$ racians

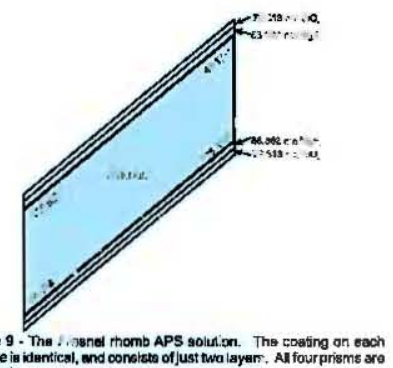
identical.

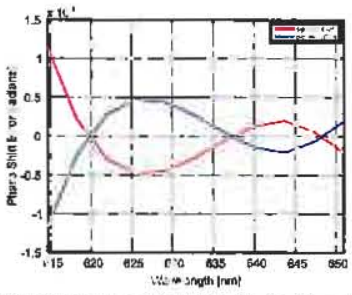

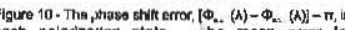
oach polarization state, he mean efror for deviation of $4.35 \times 10^{-4}$ radians. For poporizentor the masn emror is $-1.24 \times 10^{\circ}$ radians with a stendar 